\title{
Hydraulic Conductivity of Stratified Unsaturated Soils: Effects of Random Variability and Layering
}

\author{
Mohammad R. Gohardoust ${ }^{1}$, Morteza Sadeghi ${ }^{2}$, Mirkhalegh Ziatabar Ahmadi ${ }^{3}$, Scott B. Jones ${ }^{2}$, \\ and Markus Tuller ${ }^{1 *}$ \\ ${ }^{1}$ Department of Soil, Water and Environmental Science, The University of Arizona, Tucson, \\ AZ, USA \\ ${ }^{2}$ Department of Plants, Soils, and Climate, Utah State University, Logan, UT, USA \\ 3 Department Water Engineering, Sari Agricultural Sciences and Natural Resources University, \\ Sari, Iran
}

Revision 12/23/2016

Journal of Hydrology

\author{
*Corresponding Author \\ Markus Tuller \\ Professor of Soil \& Environmental Physics \\ Department of Soil, Water \& Environmental Science \\ The University of Arizona \\ Tucson, AZ 85721-0038 \\ Phone: (520) 621-7225 \\ Fax: (520) 621-1647 \\ Email: $\underline{\text { mtuller@email.arizona.edu }}$
}




\begin{abstract}
For simulating flow in heterogeneous porous media it is computationally more efficient to define an equivalent effective (i.e., upscaled) medium rather than considering detailed spatial heterogeneities. In this paper, the effective unsaturated hydraulic conductivity $(K)$ of soils exhibiting random variability, layering, or both is calculated based on numerical simulations of steady-state evaporation from a shallow water table. It is demonstrated that the effective $K$ of randomly-varied soils generally falls between the harmonic and geometric means of the unsaturated hydraulic conductivities of the constituting soils. Layering and random variability when occurring concurrently magnify each other's effects on effective $K$. As a result, the higher the degree of heterogeneity, the lower the effective $K$. Therefore, neglecting either random spatial variability or layering in numerical simulations can lead to significant overestimation of water flow in soils.
\end{abstract}

Keywords: Upscaling; Effective unsaturated hydraulic conductivity; Steady state evaporation; Soil layering; Random variability. 


\section{Introduction}

Sustainable management of subsurface water resources requires in-depth knowledge of vadose zone flow processes that are mainly governed by soil hydraulic properties (Vereecken et al. 2007). However, the commonly observed high spatial variability (e.g., random variability, layering) of soil hydraulic properties makes this a challenging task. Therefore, the characterization of spatial variability effects on soil hydraulic properties has been of great interest for decades (Miller and Miller, 1956; Willis, 1960; Warrick et al., 1985, Sharma and Luxmoore, 1979; Warrick and Yeh, 1990; Tuli et al., 2001; Khaleel et al., 2002; Zhu and Mohanty, 2002; Lu and Zhang, 2004; Assouline and Or, 2006; Schlüter et al., 2012; Sadeghi et al., 2012a; Deng and Zhu, 2015).

To improve the computational efficiency of numerical simulations and avoid potential challenges associated with detailed characterization of highly heterogeneous porous media, various upscaling approaches have been developed to estimate "effective" hydraulic properties of a hypothetically homogenous medium that is equivalent to the heterogeneous medium. Because the unsaturated hydraulic conductivity $(K)$ as a function of pressure head $(h)$ exhibits higher variability in complex heterogeneous media than the soil water characteristic, most of the proposed upscaling methods have been focused on $K$ (e.g., King, 1989; Kitanidis, 1990; Saucier, 1992; Renard and de Marsily, 1997; Neuweiler and Eichel, 2006; Neuweiler and Vogel, 2007, Samouelian et al., 2007; Hunt and Idriss, 2009).

Mualem (1984) studied the anisotropy of unsaturated layered soils considering the well-known averaging law that holds for saturated conditions (Freeze and Cherry, 1979). He stated that effective $K$ in a layered system equals to the arithmetic and harmonic mean of the individual hydraulic conductivities for flow parallel and perpendicular to the layers, respectively. Yeh et al. 
(1985) confirmed validity of this general law based on a stochastic analysis framework. However, later Yeh and Harvey (1990) challenged these previous findings illustrating that the geometric mean is a better estimate for the effective $K$ than the arithmetic or harmonic means.

Preuss (2004) further tested the applicability of the harmonic mean as effective $K$. He applied the van Genuchten (1980) $K(h)$ function to examine 1D (i.e., layering) and 2D heterogeneities and found that the harmonic mean yields reasonable approximations for unsaturated flow in relatively small domains, while the results were less accurate for larger scales. Tang et al. (2008) studied the flow characteristics of layered soils with the contour bar model based on the composite medium approximation (COMA) approach introduced by Preuss (2004). Their work was limited to layered soils with two different materials and by some means confirmed the validity of COMA for steady state conditions.

The conclusions of Preuss (2004) have been also verified by Warrick (2005) and Sadeghi et al. (2014). Based on the steady state Darcy velocity, they studied effective $K$ for several layered soil profiles consisting of repetitive layers with homogeneous sublayers of various thicknesses. They found that the effective $K$ approaches the weighted harmonic mean of the sublayer unsaturated hydraulic conductivities only when the thickness of each main layer is relatively small. Results of Sadeghi et al. (2014) indicated a nonuniform and complex relationship for the effective $K(h)$ function when sublayers exceeded a certain thickness.

Applying the Gardner (1958) $K(h)$ function, Zhu (2008) studied flow in randomly-varied soils to examine the validity of different averaging approaches for effective $K$. He concluded that for steady state vertical flow with vertical heterogeneity, the equivalent $K$ falls between the harmonic and geometric means; for coarse-textured soils and higher degrees of heterogeneity $K$ is closer to the geometric mean. Deng and Zhu (2015) investigated the anisotropy and effect of 
domain size and layer composition in two- and three-layer soils. They suggested that a proper quantification of anisotropy is required for larger flow domains, where the application of the harmonic mean would result in significant errors.

In most of the previous studies, including the work cited above, 1D heterogeneous systems were assumed to be either layered systems consisting of two or more homogeneous layers (e.g., Sadeghi et al., 2014), or a uniformly heterogeneous profile consisting of random spatial variability (e.g., Zhu, 2008). To our best knowledge, there is no study that considers both the effects of layering and random variability concurrently. Because natural layered soils also exhibit random variability, we explore effective $K(h)$ functions for such systems in this study, which is an extension of the Sadeghi et al. (2014) approach that only considered layering effects. It is demonstrated that the neglect of random variability can potentially lead to significant errors when calculating the effective $K(h)$ function for highly heterogeneous soils.

\section{Theoretical Background}

Isothermal steady state evaporation during stage II (i.e., the drying/evaporation front is below the soil surface), can be expressed with the Buckingham-Darcy law (Buckingham, 1907) considering the contributions of liquid and vapor flow to the unsaturated hydraulic conductivity:

$$
e=K\left(\frac{d h}{d z}-1\right)=\left(K_{l}+K_{v}\right)\left(\frac{d h}{d z}-1\right)
$$

where $h$ is the pressure head (the absolute value is considered for convenience), $z$ is the vertical distance from the water table (WT) to the soil surface (i.e., $z=0$ at the WT and $z=$ WT depth at the soil surface), $e$ is the steady state evaporation rate, $K_{l}$ and $K_{v}$ are the liquid and vapor hydraulic conductivities, respectively, and $K=K_{l}+K_{v}$. 
Solving for $z$, Eq. (1) yields:

$$
z=\int_{0}^{h} \frac{K(h)}{K(h)+e} d h
$$

Solution of Eq. (2) yields the pressure head distribution, $h(z)$, above the WT (see Fig. 1 in Sadeghi et al., 2014). For a known evaporation rate (e), Eq. (2) can be solved to determine the vertical distance $\left(D_{\max }\right)$ between the water table and the drying front $(\mathrm{DF})$ :

$$
D_{\text {max }}=\int_{0}^{h_{\max }} \frac{K(h)}{K(h)+e} d h
$$

where $h_{\max }$ is the pressure head at the DF.

An exact analytical solution to Eq. (3) is provided in Sadeghi et al. (2012b) for a simple power form of the $K(h)$ function. For more complex $K(h)$ functions (e.g., the van Genuchten-Mualem model), there is no exact analytical solution to Eq. (3). Nonetheless, Sadeghi et al. (2014) derived an approximate analytical solution to Eq. (3) for any arbitrary $K(h)$ function assuming:

$$
D_{\max }=h_{e}
$$

where $h_{e}$ is the pressure head at which $K=e$. Equation (4) states that pairs of $e-D_{\max }$ values coincide with the unsaturated hydraulic conductivity curve, $K(h)$, which means that the steadystate evaporation rate exhibits a measure for unsaturated hydraulic conductivity at the pressure head equal to $D_{\max }$. In summary, when $h=D_{\max }, K=e$. The applicability of this approach for coarse-textured media is due to the assumption of a symmetrical shape of $1 /[K(h)+e]$ in the derivation steps, which is not accurate for fine-textured media. Sadeghi et al. (2014) indicated that the resulting $K(h)$ curve holds for the entire $D_{\max }$ domain, which means that for 
heterogeneous soils it represents the "effective" unsaturated hydraulic conductivity of the entire heterogeneous profile. Therefore, this method provides a unique opportunity to directly calculate the effective $K$ curve for various arbitrary heterogeneous soil profiles via forward steady-state simulations based on the Buckingham-Darcy law (Buckingham, 1907). This approach is computationally more efficient and much simpler than the conventional inverse solution approach based on Richards' equation (Richards, 1931). In addition, this new method is not restricted to a specific mathematical form of the $K(h)$ function, and therefore more appropriate than the inverse solution for layered soils, where the effective $K(h)$ curve may substantially differ from the conventional unimodal $K(h)$ functions (Sadeghi et al., 2014).

\section{Materials and Methods}

\subsection{Investigated Heterogeneous Soil Profiles}

In this study, 1D heterogeneity perpendicular to the flow direction has been considered. Effective $K(h)$ functions for one-, two-, and three-layer soil profiles were numerically simulated based on the Sadeghi et al. (2014) approach. For each layer, a distinct soil was used as reference. Each layer contained numerous increments of $0.1-\mathrm{cm}$ thickness each with randomly-varied hydraulic properties. Hence, the total number of increments was variable depending on the extent of the liquid flow domain above the WT (i.e., $D_{\max }$ ).

The van Genuchten (VG) (1980) hydraulic conductivity model was applied:

$$
K_{l}=K_{S} \frac{\left\{1-(\alpha h)^{n-1}\left[1+(\alpha h)^{n}\right]^{-m}\right\}^{2}}{\left[1+(\alpha h)^{n}\right]^{m / 2}}
$$


where $\alpha, n$, and $m$ are empirical model parameters with the assumption $m=1-1 / n$. Note that $K_{v}$ was not considered in this study, because the analysis was limited to the liquid flow range following Sadeghi et al. (2014).

Four coarse-textured soils with VG parameters listed in Table 1 were selected from literature as reference soils; Sand-1 from Smits et al. (2012), Loveland Sand from Anat (1965), Hygiene Sandstone from van Genuchten (1980), and Packed Sand from Minasny et al. (2004).

\section{$\underline{\text { Insert Table } 1}$}

Miller and Miller (1956) similar-media scaling theory was applied to generate random variability for each increment by considering randomly-generated scaling factors. A lognormal distribution was used for generation of the random scaling factors. This assumption is based on actual field observations (e.g., Warrick et al., 1977; Kosugi and Hopmans, 1998) indicating that the spatial variability of soil hydraulic properties can be adequately represented by a single stochastic scaling factor, which commonly exhibits a lognormal distribution.

Assuming validity of the Miller and Miller (1956) theory, Sadeghi et al. (2016) related the Miller and Miller scaling factor to the scale parameters of common unimodal soil hydraulic functions. The relationships for the VG model are given as:

$$
\alpha_{i}=\lambda_{i} \alpha_{R}, K_{s_{i}}=\lambda_{i}^{2} K_{s_{R}}, n_{i}=n_{R}
$$

where $\lambda$ is the randomly generated scaling factor for each increment, and subscripts $R$ and $i$ denote the reference soil and soil at the increment $i$, respectively. This means that greater scaling factors increase the saturated hydraulic conductivity and shift the air entry pressure value closer 
to zero, while the slope of the $K(h)$ curve remains unchanged. This mimics a pore size distribution with higher mean but the same standard deviation.

For the one-layer scenario, 4 one-layer profiles corresponding to the 4 reference soils (Table 1 ) were studied. All the increments were assumed to be randomly-varied via the random scaling factors and related to the reference soil through Eq. (6). The lognormal distributions applied for generation of the scaling factors were assumed to have a mean of 0 (Warrick et al., 1977; Kosugi and Hopmans, 1998) and standard deviations (SD) of 0.01 (i.e., negligible variability) and 0.1 (i.e., significant variability). The "lognrnd" function embedded in the Matlab 8.0 statistics toolbox (MathWorks, Inc., Natick, MA, USA) was applied for generation of the random scaling factors. It should be noted that the randomness pattern was kept the same for all one-layer columns.

For the two- and three-layer scenarios, we considered one specific layering for each scenario. For the two-layer scenario, a 40-cm layer of Loveland Sand on top of a 23-cm Sand-1 layer was considered. For the three-layer system, we used 100-cm Packed Sand on top of 15 and 20-cm Loveland Sand and Sand-1 layers, respectively. The random variability within each layer for both the two- and three-layer scenarios was also considered. Note that the layer thicknesses were chosen such that the relationship between effective $K(h)$ and $K(h)$ for all individual increments could be visually discerned in the graphs.

\subsection{Numerical Simulations}

Steady state evaporation for the heterogeneous profiles discussed above was numerically simulated with Eqs. (3), (5) and (6) with $e$ as an arbitrary input parameter. $D_{\max }(e)$ as an approximation for $h(K)$ was calculated based on the following pseudocode (see also Fig. 1): 
I. $\quad$ Let $i=0$,

II. Calculate $h_{i+1}$ such that the following equation holds $\left(h_{0}=0\right)$ :

$$
z_{i+1}-z_{i}=\Delta z=\int_{h_{i}}^{h_{i+1}} \frac{K_{i}}{K_{i}+e} d h
$$

III. Let $i=i+1$,

IV. Repeat steps II and III until either $h_{i+1}>h_{\max }$ (i.e., $h$ at which $K_{l}=K_{v}$ ) or Eq. (7) does not converge for $h_{i+1}$ (this occurs for the final $\Delta z$ because $d h / d z \rightarrow \infty$ ).

V. Calculate $\delta$ as:

$$
\delta=\int_{h_{i}}^{h_{\max }} \frac{K_{i+1}}{K_{i+1}+e} d h
$$

VI. Calculate $D_{\max }$ as:

$D_{\max }=z_{i}+\delta$

\section{$\underline{\text { Insert Figure } 1}$}

Note that the integrals in Eqs. (7) and (8) were solved with the "fzero" function in Matlab 8.0. The evaporation rate $(e)$ was considered as an arbitrary input and kept constant for each individual simulation. However, simulations were performed for a wide range of $e$ values to yield the effective $K$ within that range. Sadeghi et al. (2014) suggested the upper limit of $e$ applicable to homogeneous soils to be two orders of magnitude lower than $K_{s}$. Here we considered the limit to be two orders of magnitude lower than the geometric mean of $K_{s}$ of all increments. The lower limit of $e$ was assumed to coincide with $10^{-7} \mathrm{~cm} \mathrm{~d}^{-1}$, because the proposed 
analysis applies only to the liquid flow domain. According to the $K\left(h_{\max }\right)$ values of the reference soils listed in Table 2, the lower limit falls within the liquid flow domain for all soils.

\section{$\underline{\text { Insert Table } 2}$}

\section{Results and Discussion}

\subsection{Random Variability Effects}

Simulation results for the 4 one-layer soil columns are depicted in Figs. 2 and 3 for scaling factor standard deviations of 0.01 (i.e., negligible random variability) and 0.1 (i.e., significant random variability). The effective $K-h$ data are equivalent to the $e-D_{\max }$ pairs obtained from the numerical simulations. It is obvious that the Sadeghi et al. (2014) approach not only enables estimation of effective unsaturated hydraulic conductivity, but also allows quantification of the effect of each increment's variability on the effective $K(h)$ curve. The former is evident from Fig. 2, which shows that the $e-D_{\max }$ pairs (effective $K$ in the plots) coincide with the reference $K(h)$ curve, and the latter is exhibited in Fig. 3.

Figure 3 clearly shows that the effects of random variability on effective $K$ investigated here is different from the effects of periodic variability studied previously (e.g. Warrick, 2005; Neuweiler and Eichel, 2006; Zhu and Warrick, 2012; Sadeghi et al., 2014). For cases of periodic variability with extremely small increments, the effective $K$ approaches the harmonic mean of the individual $K$ curves. This is contradicted by results for random variability depicted in Fig. 3 that indicate that effective $K$ falls between the geometric and harmonic mean even though the length increments are very small (i.e. $0.1 \mathrm{~cm}$ ). Note that in natural systems the occurrence of random variability is more likely than periodic variability. 
Zhu (2008) reported similar findings based on a general $p$-norm averaging scheme that was introduced by Journel et al. (1986) with an exponent $p$ ranging from -1 to 1 such that $p=1,0$, and -1 represent the arithmetic, geometric and harmonic means, respectively. Our results are more general than results reported in $Z h u$ (2008). We applied the more realistic VG model (our approach is generally amenable to any arbitrary $\mathrm{K}(\mathrm{h})$ function), while $Z$ hu's findings are based on the application of the Gardner (1958) exponential hydraulic conductivity function. Furthermore, Zhu (2008) only reported the fitting averaging power $(-1<p<0)$, while the detailed effective $K(h)$ relationship exhibited in Fig. 3 cannot be obtained with the $p$-norm averaging scheme. This demonstrates the utility of Sadeghi et al. (2014) approach, which has its own limitations for coarse-textured soils as discussed in Lehmann et al. (2015) and Sadeghi et al. (2015).

\section{$\underline{\text { Insert Figure } 2}$}

\section{$\underline{\text { Insert Figure } 3}$}

Results of this study reveal a close relationship between the hydraulic properties of each increment and the resultant change in the effective $K$ in that increment. As depicted in Fig. 4, the patterns of the spatial distribution of the scaling factors along the soil profile and the patterns of the slope of the effective $K(h)$ curve on the logarithmic scale are similar, especially when the SD is small. The slope was quantified by the angle of the curve relative to the horizon within the range $[-\pi / 2,0]$, where $-\pi / 2$ and 0 correspond to vertical and horizontal lines, respectively. It should be noted that the effective $K(h)$ curves in Figs. 2 and 3 resulted from the specific order of random increments, and hence, are associated with one of many possible realizations.

It is evident that increments with scaling factors larger than 1 (corresponding to coarser soils) tend to increase the slope of the effective $K$ curve, which means a higher rate of decrease of 
effective $K$ per unit increase of pressure head. On the other hand, increments with scaling factors smaller than 1 push the effective $K$ curve towards the vertical line, which translates to a lower rate of decrease of the effective $K$ per unit increase of pressure head. This trend becomes biased when the SD (i.e., random variability) increases, because a more vertical slope due to any fine increment propagates through many increments, while a more horizontal slope hardly spans more than a few increments. Coarser increments play an important role here as a highly negative slope (a jump) occurs right after any coarse increment (i.e., plateaus observed in the effective $K(h)$ curve). A pronounced example for such case is the observed jump around $K=10 \mathrm{~cm} \mathrm{~d}^{-1}$ for Sand 1 in Fig. 3.

\section{Insert Figure 4}

As shown in Figs. 2 and 3, the VG model was fitted to the effective $K(h)$ curve to more specifically determine where the effective curve falls between the reference soil and harmonic mean curves. The fitted VG curve was obtained with a fitting scaling factor, $\lambda_{\text {eff. }}$ Similarly, a scaling factor $\lambda_{h m}$ best describing the harmonic mean curve was calculated for each soil profile. Note that the geometric mean coincides with the reference curve (i.e., $\lambda=1$ ).

Figure 5 depicts the obtained $\lambda_{\text {eff }}$ and $\lambda_{h m}$ scaling factors for the 4 one-layer profiles. The range of the SD of the lognormal distributions was extended beyond 0.1 to better visualize the trend. Figure 5 shows that the effective $K$ curve is almost centered between the geometric and harmonic means for the Loveland Sand and Hygiene Sandstone, while it is closer to the harmonic mean for Sand-1 and Packed Sand. This finding somewhat contradicts Zhu (2008), who concluded that "for coarse-textured soils and a more heterogeneous landscape, the equivalent hydraulic conductivity should move more towards the geometric mean". This comparison further emphasizes the importance of the variability structure and also the interrelation between $\alpha$ and $K_{s}$ 
as also highlighted in Zhu (2008). While the Miller and Miller type variability studied here implicitly assumes a perfect correlation $(r$-squared $=1)$ between $\alpha$ and $K_{s}($ Eq. $(6))$, Zhu (2008) considered an imperfect correlation $(r$-squared $<1)$ between $\alpha$ and $K_{s}$. An evaluation of the validity of the Miller and Miller scaling theory and the interrelation between $\alpha$ and $K_{s}$ for natural soils in Sadeghi et al. (2016) indicated that the perfect correlation between $\alpha$ and $K_{s}$ does not necessarily hold in reality, and hence, the simultaneous scaling (i.e., equality of scaling factors for $h$ and $K$, Eq. (6)) is not always a valid assumption.

\section{$\underline{\text { Insert Figure } 5}$}

\subsection{Concurrent Random Variability and Layering Effects}

Figure 6 depicts concurrent effects of layering and random variability on the effective $K$ curve for the two- and three-layer soil profiles. The sole effect of layering is evident for a SD $=0.01$ (i.e., negligible random variability). As observed here and concluded in Sadeghi et al. (2014), the layering effect can be generalized as "Each layer's individual curve contributes to the effective curve at hequal to the distance of the layer to the water table and the effective curve transitions from the individual curve of the bottom layer to the individual curve of the top layer when $h$ exceeds the distance of the layer interface to the water table" (see Fig. 10 in Sadeghi et al., 2014). When random variability is added (i.e. $\mathrm{SD}=0.1$ ), the effective curve shifts downwards and appears noisier.

A close inspection of the $\lambda_{\text {eff }}$ scaling factors obtained for the two-layer profile reveals that $\lambda_{\text {eff }}$ for the bottom layer (Sand-1) remains almost unchanged when compared to the one-layer Sand-1 profile, while $\lambda_{\text {eff }}$ of the top layer (Loveland Sand) is larger than $\lambda_{\text {eff }}$ of the Loveland Sand onelayer profile. This moves the position of the effective $K$ curve closer to the harmonic mean. As 
evident from Fig. 6, this trend is more pronounced for the lower increments of the top layer (i.e. increments closer to the interface). This increase in $\lambda_{\text {eff }}$ is also observed for the middle and the upper layers of the three-layer profile. To explore the generality of this behavior, we tested a few different arrangements of the random sublayers and observed the same behavior for all arrangements.

These observations indicate that layering not only affects the effective $K$ on its own, but also magnifies the random variability effect (i.e. shifting the effective curve further down). This means that the higher the degree of heterogeneity, the lower the effective conductivity. Therefore, neglecting either random spatial variability or layering in numerical simulations can lead to significant overestimation of water flow in soils. This is in agreement with findings of Botros et al. (2012).

\section{$\underline{\text { Insert Figure } 6}$}

\subsection{Comparison with the Simplified Evaporation Method}

It is well documented that, regardless of the type of the upscaling method, the estimated effective properties depend not only on the properties of the constituent materials, but also on the boundary conditions (Vereecken et al., 2007). Therefore, the effective $K$ curves presented in this study are not necessarily valid for other flow processes such as infiltration or transient evaporation. This limitation is inevitable for layered profiles as it is not possible to define a "representative elementary volume" (REV) descriptive for the entire soil profile. For example, if we compare infiltration with evaporation from a deep groundwater table in a deep two-layer profile, the effective $K$ for the infiltration process is equivalent to the $K$ of the surface layer provided that it is thick enough, while the effective $K$ for the evaporation process is equivalent to 
the $K$ of the bottom layer provided that the groundwater table is deep enough (i.e., the bottom layer encompasses the entire liquid flow domain).

Unlike for layered soils, this restriction may not apply to soils with random spatial variability, because a REV can be usually defined for such soils (i.e., the heterogeneity structure is similar along the profile); hence the effective $K$ obtained with the steady-state evaporation method might be valid for other flow processes besides steady state evaporation, which is investigated in the following.

To evaluate the applicability of effective $K$ for transient evaporation, the simplified evaporation method (SEM) (Peters and Durner, 2008) was applied to estimate the hydraulic properties of the 4 randomly-varied one-layer profiles described above. The SEM is commonly applied for measuring the water characteristic and hydraulic conductivity curve in the laboratory. A 6-cm tall soil sample with 2 miniature tensiometers installed in 1.5 and $4.5-\mathrm{cm}$ depths is commonly employed for this experiment. The soil column is first saturated and then exposed to the atmosphere for water to evaporate from the surface, while the change of mass (i.e. evaporation rate) is continuously recorded throughout the experiment. The soil water characteristic is obtained from the relationship between the average water content and the average pressure head known from the tensiometer readings. The unsaturated hydraulic conductivity curve is estimated from the relationship between evaporation rate and pressure head gradient based on the Buckingham-Darcy law (Buckingham, 1907).

We applied the HYDRUS-1D numerical code (Simunek et al., 2008) to simulate transient evaporation from 6-cm tall soil columns randomly sampled from the studied randomly-varied soil profiles. For each soil we performed two evaporation simulations, one for $\mathrm{SD}=0.01$ and one 
for $\mathrm{SD}=0.1$. The $K(h)$ curve of the samples was then estimated with the SEM based on simulated values for average water content, tensiometer readings and evaporation rate.

Figure 7 depicts a comparison of $K(h)$ curves obtained with the SEM and the effective $K(h)$ curve for the entire one-layer profiles obtained with the steady state method (Sand-1 has been excluded due to instabilities of numerical results for such a coarse and heterogeneous medium). Though there are evident discrepancies for Hygiene Sandstone, $K(h)$ curves obtained with both methods compare reasonably well. These findings postulate that the effective $K(h)$ curve for a random variability structure is relatively invariant with respect to boundary conditions and thus it can be applied for other flow processes besides steady state evaporation. The $K(h)$ curve obtained with the steady-state method is smoother than the curve of the SEM, especially for the case of $\mathrm{SD}=0.01$. This is mainly attributable to the much larger sample investigated with the steady state method. According to homogenization theory, a soil sample can be assumed as homogenous if the sample size is much larger than the scale of the heterogeneity structure (Neuweiler and Eichel, 2006).

\section{Insert Figure 7}

\subsection{Limitations due to $1 D$ Heterogeneity}

All analyses in this paper are based on the assumption of a 1D heterogeneity structure. In other words, the presented effective $K(h)$ curves are valid only for their respective 1D realizations with the specific order of random sublayers. A 1D structure rarely occurs in nature and 2D and 3D patterns would be more realistic. Considering that $2 \mathrm{D}$ and $3 \mathrm{D}$ media include many $1 \mathrm{D}$ realizations, it can be intuitively predicted that the steady-state evaporation method should result in smoother effective $K(h)$ curves from 2D and 3D simulations. As an example, average effective 
$K(h)$ for 50 different realizations of random 1D patterns for Sand-1 and Loveland sand is depicted in Fig. 8. We would expect similar results from 2D and 3D simulations as shown in Fig. 8, provided that lateral flow in negligible. Comparing Figs. 8 and 3, we predict that the location of the effective curve (i.e., $\lambda_{\text {eff }}$ ) would not be significantly different between 1-, 2- and 3dimensional heterogeneity structures. The effective $K(h)$ curve would, however, be smoother for higher dimensions.

\section{$\underline{\text { Insert Figure } 8}$}

\section{Summary and Conclusions}

It has been demonstrated that the Sadeghi et al. (2014) steady state evaporation method enables estimation of the effective unsaturated hydraulic conductivity of randomly-varied and layered soils. Based on the forgoing analyses we conclude:

I. The effective $K$ is sensitive to the heterogeneity structure. For example, the effect of random variability on the effective $K$ is different from effects of periodic variability. In case of periodic variability with extremely small increments, the effective $K$ approaches the harmonic mean of the individual $K$ curves, while the effective $K$ of randomly-varied soils falls between the geometric and harmonic means.

II. There is an intimate relationship between the hydraulic properties of each increment and the shape of the effective $K(h)$ curve. An increment with higher scaling factor tends to incline the effective $K$ to a horizontal line at $h$ equal to the thickness of the specific increment, while increments with lower scaling factors pushes the effective $K$ curve towards the vertical line. 
III. Layering and random variability when occurring concurrently magnify each other's effects on effective $K$. When random variability is added to a layered profile, the effective $K(h)$ curve is shifted downwards. In addition, layering affects the effective $K(h)$ curve not only on its own, but also magnifies the random variability effect to further decrease effective $K$. In other words, the higher the degree of heterogeneity, the lower the effective $K$. Therefore, neglecting either random spatial variability or layering in numerical simulations leads to significant overestimation of water flow in soils.

$I V$. The effective $K$ estimated based on the steady state method is a process-dependent property of layered systems (i.e., no REV). However, the effective $K$ is process-independent for randomly-varied soils for which a REV can be defined and thus can be applied for other flow processes besides steady state evaporation. Here we only examined transient evaporation but further studies are required to assess the validity of this claim for other scenarios such as infiltration.

The application of the presented approach to study effective hydraulic conductivity of $2 \mathrm{D}$ and $3 \mathrm{D}$ heterogeneity structures is part of ongoing research.

\section{Acknowledgements}

The authors gratefully acknowledge support from the National Science Foundation (NSF) through grant no. 1521469, awarded to Utah State University and the University of Arizona, and from the Arizona Agricultural Experiment Station (AAES). 


\section{References}

Anat, A., 1965. Steady upward flow from water tables. Ph.D. dissertation. Colorado State University, Fort Collins, Colorado.

Assouline, S., Or, D., 2006. Anisotropy factor of saturated and unsaturated soils. Water Resour. Res. 42, W12403.

Botros, F.E., Onsoy, Y.S., Gin, T.R., Harter, T., 2012. Richards Equation-Based Modeling to Estimate Flow and Nitrate Transport in a Deep Alluvial Vadose Zone. Vadose Zone J., 11(4), doi:10.2136/vzj2011.0145.

Buckingham, E., 1907. Studies on the movement of soil moisture. Bull., USDA, Bureau of Soils, vol. 38. Washington D.C. 
Deng, P. and Zhu, J., 2015. Anisotropy of Unsaturated Layered Soils: Impact of Layer Composition and Domain Size. Soil Sci. Soc. Am. J. 79(2), 487-494.

Freeze, R.A., and Cherry, J.A., 1979. Groundwater. Englewood Cliffs, NJ, Prentice-Hall, 604 p.

Gardner, W.R., 1958. Some steady-state solutions of the unsaturated moisture flow with applications to evaporation from a water table. Soil Sci. 85(4), 28-232.

Hunt, A.G., Idriss, B., 2009. Percolation-based effective conductivity calculations for bimodal distributions of local conductances. Philos. Mag. 89, 22-24.

Journel, A.G., Deutsch, C., Desbarats, A.J., 1986. January. Power averaging for block effective permeability. SPE California Regional Meeting. Society of Petroleum Engineers.

Khaleel, R., Yeh, T.-C.J., Lu, Z., 2002. Upscaled flow and transport properties for heterogeneous unsaturated media. Water Resour. Res. 38(5), 1053.

King, P.R., 1989. The use of renormalization for calculating effective permeability. Transp. Porous Media. 4, 37-58.

Kitanidis, P.K., 1990. Effective hydraulic conductivity for gradually varying flow. Water Resour. Res. 26, 1197-1208.

Kosugi, K., Hopmans, J.W., 1998. Scaling water retention curves for soils with lognormal poresize distribution. Soil Sci. Soc. Am. J., 62, 1496-1504.

Lehmann, P., Assouline, S., Or, D., 2015. Comment on "Column-scale unsaturated hydraulic conductivity estimates in coarse-textured homogeneous and layered soils derived under steady-state evaporation from a water table” by M. Sadeghi, M. Tuller, M.R. Gohardoust and S.B. Jones. J. Hydrol. 529: 1274-1276.

Lu, Z., Zhang, D., 2004. Analytical solutions to steady state unsaturated flow in layered, randomly heterogeneous soils via Kirchhoff transformation. Adv. Water Resour. 27, 775784. 
Miller E.E., and Miller R.D. 1956. Physical theory for capillary flow phenomena. Journal of Applied Physics, 27: 324-332.

Minasny, B., Hopmans, J., Harter, T., Eching, S.O., Tuli, A., Denton, M.A., 2004. Neural networks prediction of soil hydraulic functions for alluvial soils using multistep outflow data. Soil Sci. Soc. Am. J. 68, 417-429.

Mualem, Y., 1984. Anisotropy of Unsaturated Soils. Soil Sci. Soc. Am. J. 48, 505-509.

Neuweiler, I., Eichel, H., 2006. Effective parameter functions for Richards equation in layered porous media, Vadose Zone J. 5, 963-977.

Neuweiler, I., Vogel, H.-J., 2007. Upscaling for unsaturated flow for non-Gaussian heterogeneous porous media. Water Resour. Res. 43, W03443.

Peters, A., Durner, W., 2008. A simple model for describing hydraulic conductivity in unsaturated porous media accounting for film and capillary flow. Water Resour. Res. 44, W11417.

Pruess, K., 2004. A composite medium approximation for unsaturated fl ow in layered sediments. J. Contam. Hydrol. 70, 225-247.

Renard, P., De Marsily, G., 1997. Calculating equivalent permeability: a review. Adv. Water Resour. 20, 253-278.

Richards, L.A., 1931. Capillary conduction of liquids through porous mediums. J. Appl. Phys. 1, 318-333.

Sadeghi, M., Ghahraman, B., Warrick, A.W., Tuller, M., Jones, S.B., 2016. A critical evaluation of the Miller and Miller similar media theory for application to natural soils. Water Resour. Res. 52, 3829-3846.

Sadeghi, M., Ghahraman, B., Ziaei, A.N., Davary, K., Reichardt, K., 2012a. Invariant solutions of Richards' equation for water movement in dissimilar soils. Soil Sci. Soc. Am. J. 76, 1-9. 
Sadeghi, M., Shokri, N., Jones, S.B., 2012b. A novel analytical solution to steady-state evaporation from porous media. Water Resour. Res. 48, W09516.

Sadeghi, M., Tuller, M., Gohardoust, M.R., Jones, S.B., 2014. Column-scale unsaturated hydraulic conductivity estimates in coarse-textured homogeneous and layered soils derived under steady-state evaporation from a water table. J. Hydrol. 519, 1238-1248.

Sadeghi, M., Tuller, M., Gohardoust, M.R., Jones, S.B., 2015. Reply to comments on “'Columnscale unsaturated hydraulic conductivity estimates in coarse-textured homogeneous and layered soils derived under steady-state evaporation from a water table" [J. Hydrol. 519 (2014), 1238-1248]. J. Hydrol. 529, 1277-1281.

Samouelian, A., Vogel, H.J., Ippisch, O., 2007. Upscaling hydraulic conductivity based on the topology of the sub-scale structure. Adv. Water Resour. 30, 1179-1189.

Saucier, A., 1992. Effective permeability of multifractal porous media. Physica A. 183, 381-397.

Sharma, M.L., Luxmoore, R.J., 1979. Soil spatial variability and its consequence on simulated water balance. Water Resour. Res. 15, 1567-1573.

Schlüter, S., Vanderborght, J., Vogel, H.-J., 2012. Hydraulic non-equilibrium during infiltration induced by structural connectivity. Adv. Water Resour., 44, 101-112.

Simunek, J., Sejna, M., Saito, H., Sakai, M., van Genuchten, M.Th., 2008. The HYDRUS-1D software package for simulating the one-dimensional movement of water, heat, and multiple solutes in variably-saturated media, Version 4.0., University of California, Riverside, CA.

Smits, K.M., Ngo, V.V., Cihan, A., Sakaki, T., Illangasekare, T.H., 2012. An evaluation of models of bare soil evaporation formulated with different land surface boundary conditions and assumptions. Water Resour. Res. 48, W12526.

Tang, G., Perfect, E., van den Berg, E.H., Mayes, M.A., Parker, J.C., 2008. Estimating effective hydraulic parameters of unsaturated layered sediments using a Cantor bar composite medium model. Vadose Zone J. 7, 493-499. 
Tuli, A., Kosugi, K., Hopmans, J.W., 2001. Simultaneous scaling of soil water retention and unsaturated hydraulic conductivity functions assuming lognormal pore-size distribution. Adv. Water Resour. 24, 677-688.

van Genuchten, M.T., 1980. A closed-form equation for predicting the hydraulic conductivity of unsaturated soils. Soil Sci. Soc. Am. J. 44, 892-898.

Vereecken, H., Kasteel, R., Vanderborght, J., Harter, T., 2007. Upscaling hydraulic properties and soil water flow processes in heterogeneous soils: a review. Vadose Zone J. 6, 1-28.

Warrick, A.W., 2005. Effective unsaturated hydraulic conductivity for one-dimensional structured heterogeneity. Water Resour. Res. 41, W09406.

Warrick, A.W., Lomen, D.O., Yates, S.R., 1985. A generalized solution to infiltration, Soil Sci. Soc. Am. J. 49, 34-38.

Warrick, A.W., Yeh, T.C.J., 1990. One-dimensional, steady vertical flow in a layered soil profile. Adv. Water Resour. 13, 207-210.

Warrick A.W., Mullen, G.J., Nielsen, D.R., 1977. Scaling of field measured hydraulic properties using a similar media concept, Water Resour. Res. 13, 355-362.

Willis, W.O., 1960. Evaporation from layered soils in the presence of a water table. Soil Sci. Soc. Am. Proc. 24, 239-242.

Yeh, T.-C.J., Gelhar, L.W., Gutjahr, A.L., 1985. Stochastic analysis of unsaturated flow in heterogeneous soils: 2 . Statistically anisotropic media with variable $\alpha$. Water Resour. Res. $21,457-464$.

Yeh, T.-C.J., Harvey, D.J., 1990. Eff ective unsaturated hydraulic conductivity of layered sands. Water Resour. Res. 26, 1271-1279.

Zhu, J., Mohanty, B.P., 2002. Spatial averaging of van Genuchten hydraulic parameters for steady-state flow in heterogeneous soils: A numerical study. Vadose Zone J. 1, 261-272. 
Zhu, J., 2008. Equivalent parallel and perpendicular unsaturated hydraulic conductivities: Arithmetic mean or harmonic mean? Soil Sci. Soc. Am. J. 72(5), 1226-1233.

Zhu, J. and Warrick, A.W., 2012. Unsaturated Hydraulic Conductivity of Repeatedly Layered Soil Structures. Soil Sci. Soc. Am. J. 76(1), 28-35. 
$z_{i+1}=(i+1) \Delta z \longrightarrow h_{i+1}$

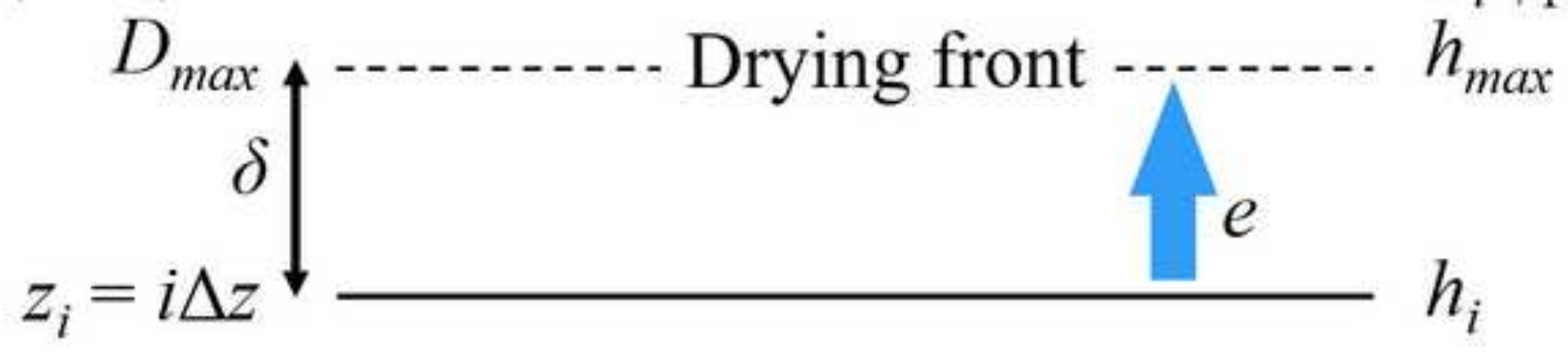

$\lambda_{i} \alpha_{R}, \lambda_{i}^{2} K_{s_{R}}, n_{R}$

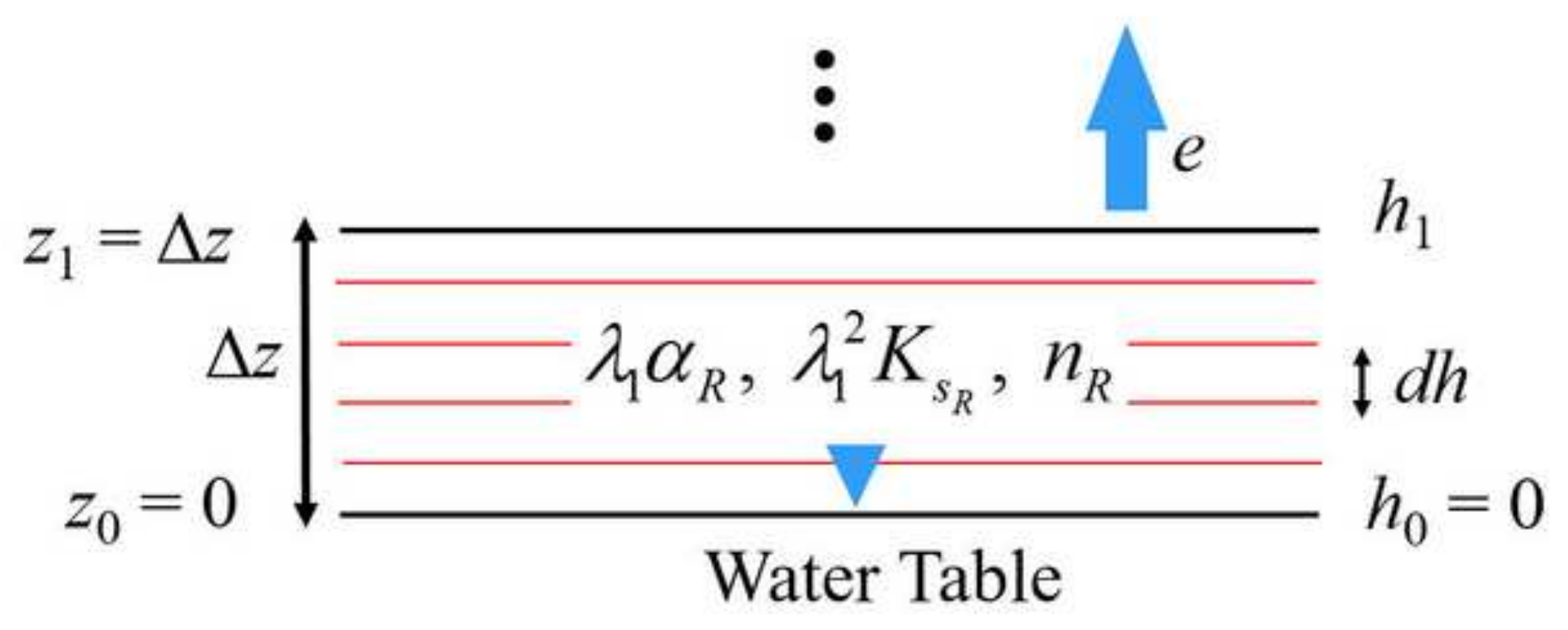



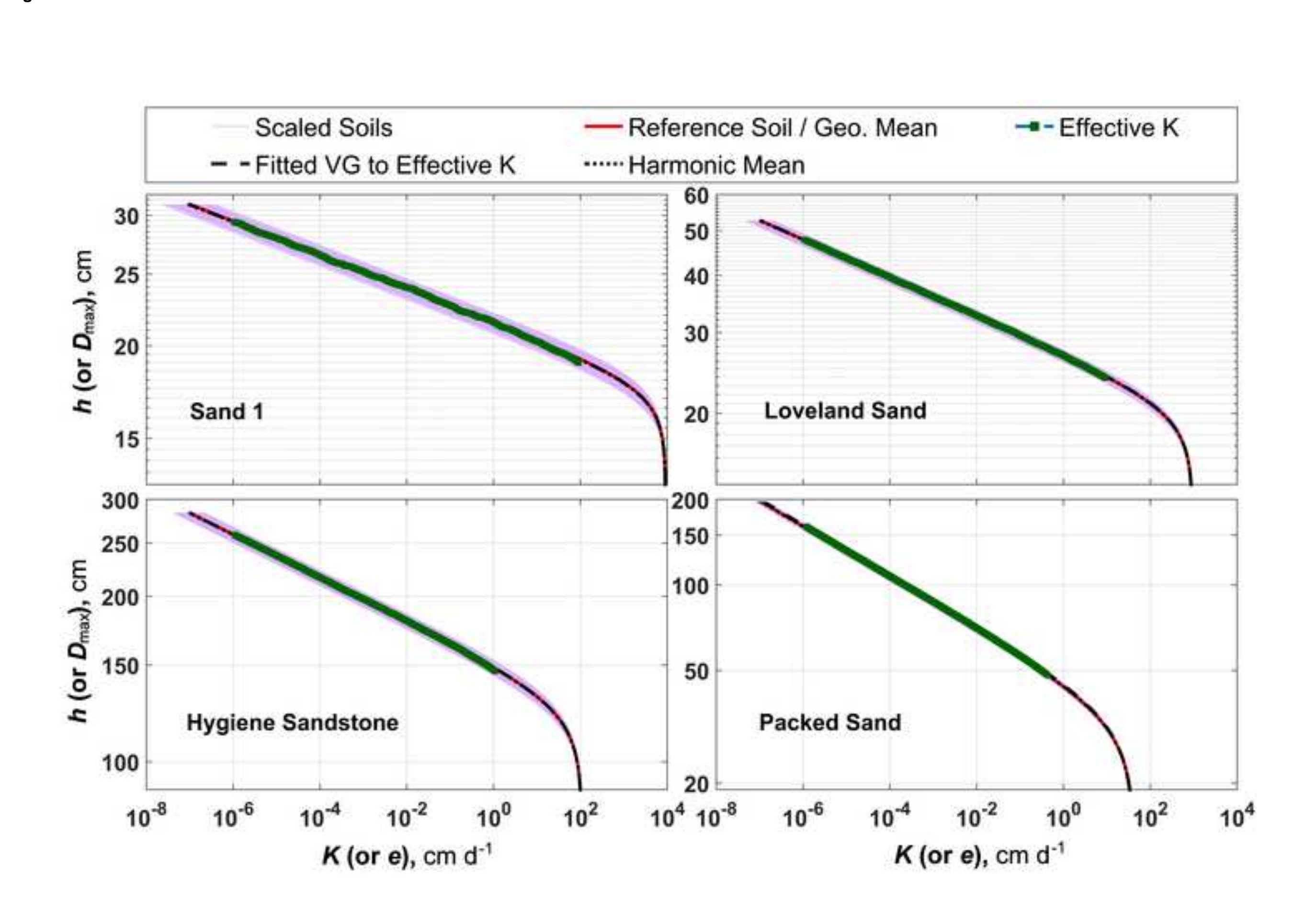

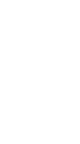

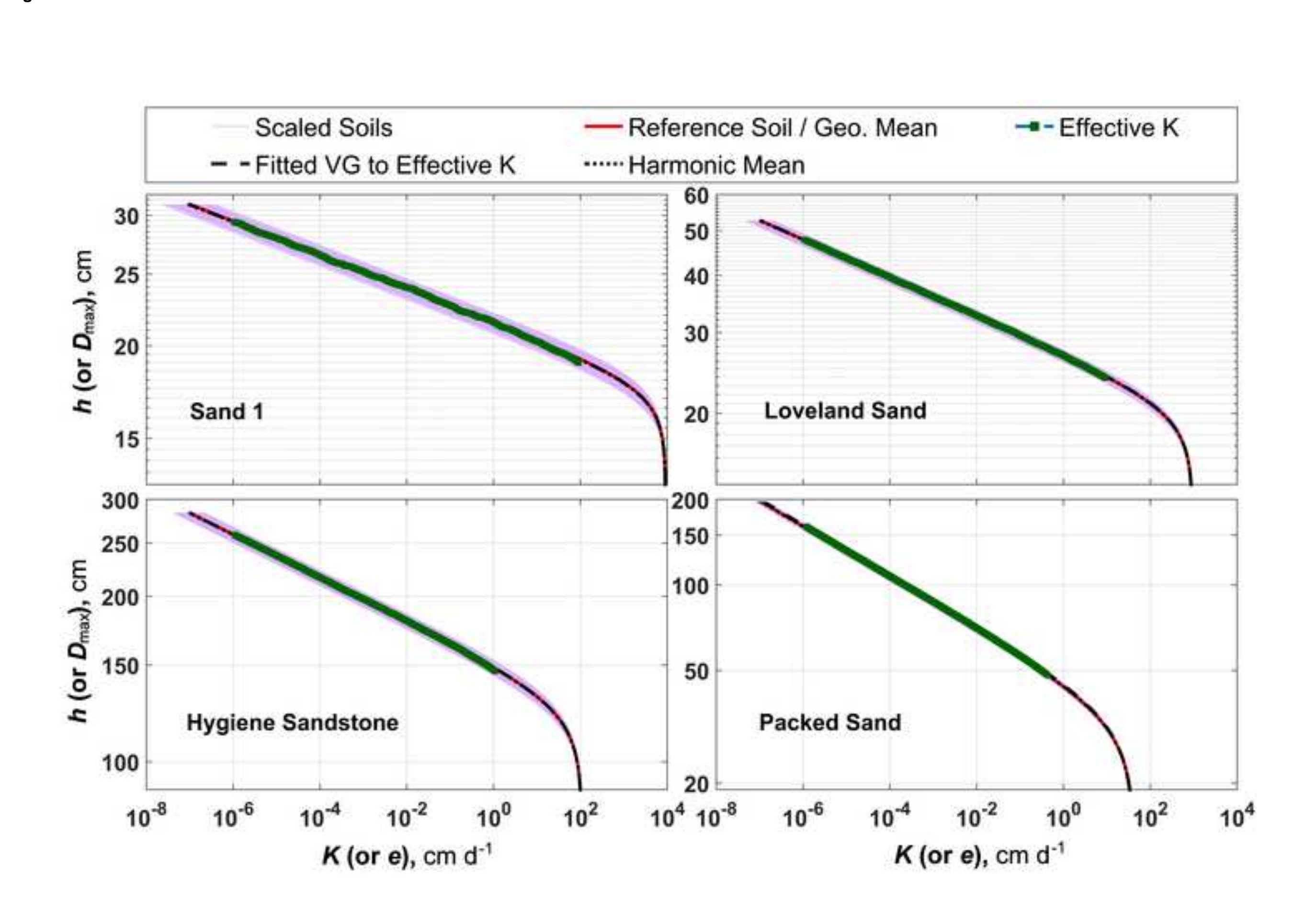

.

.

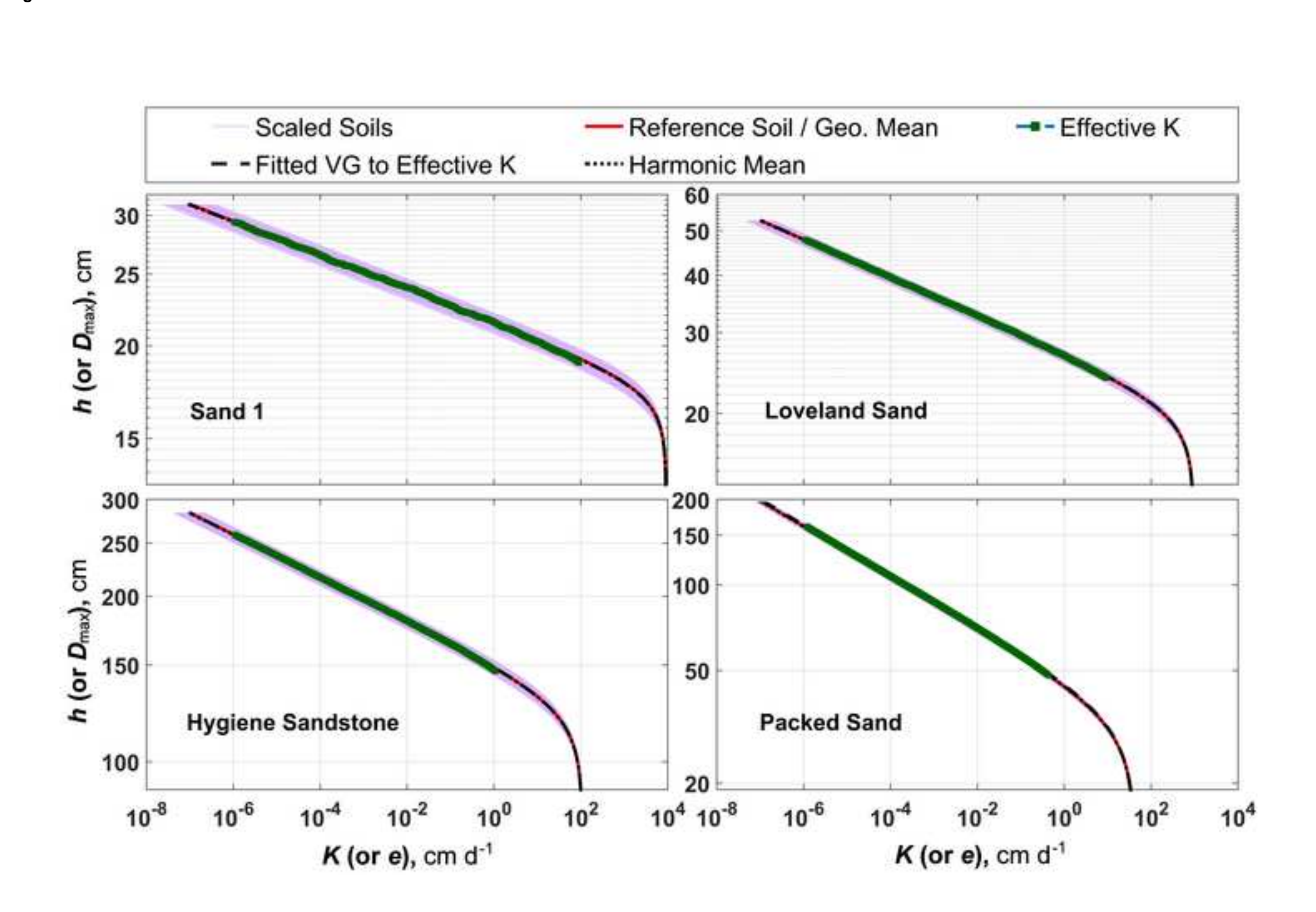

.

.

.

.

.

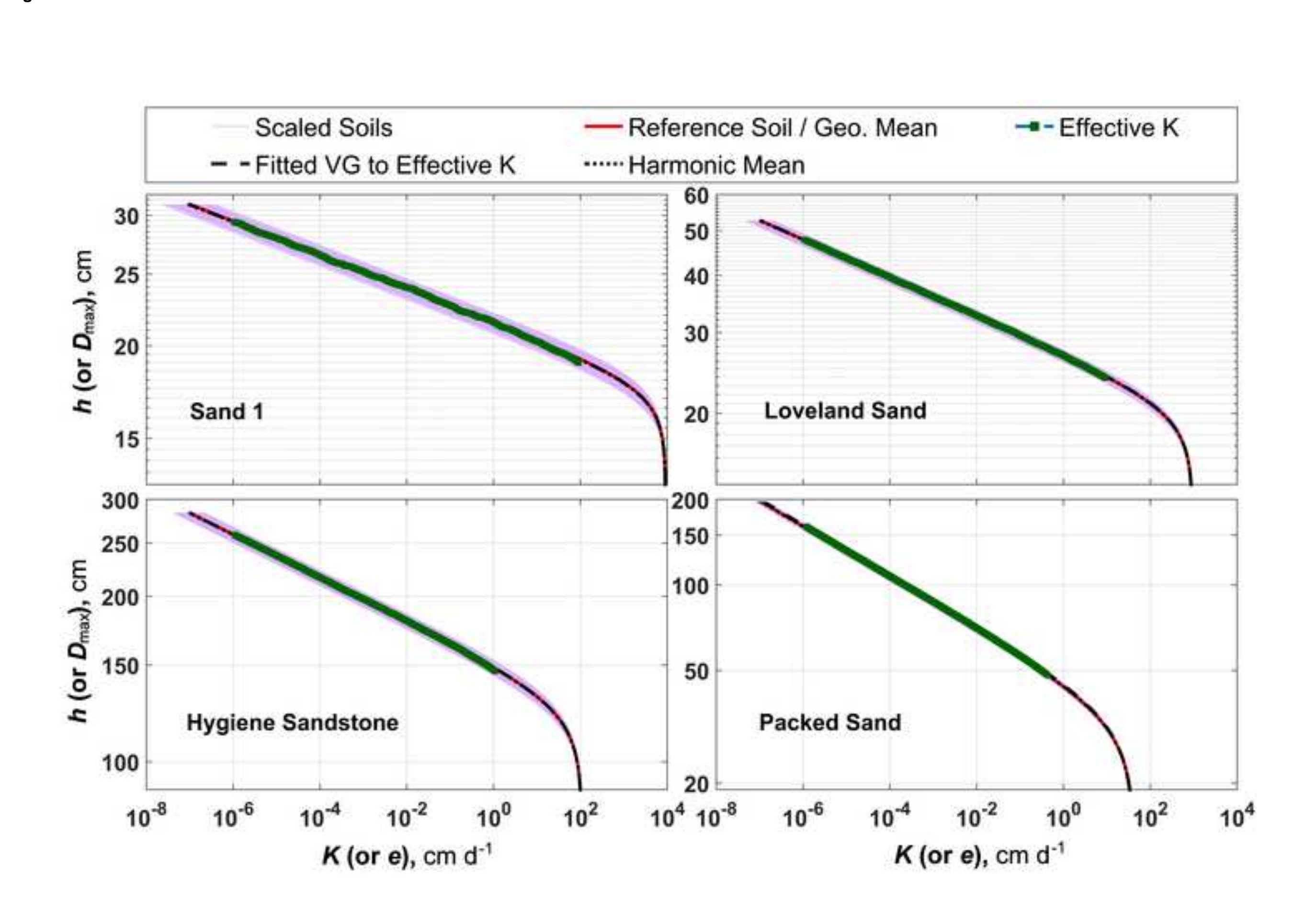




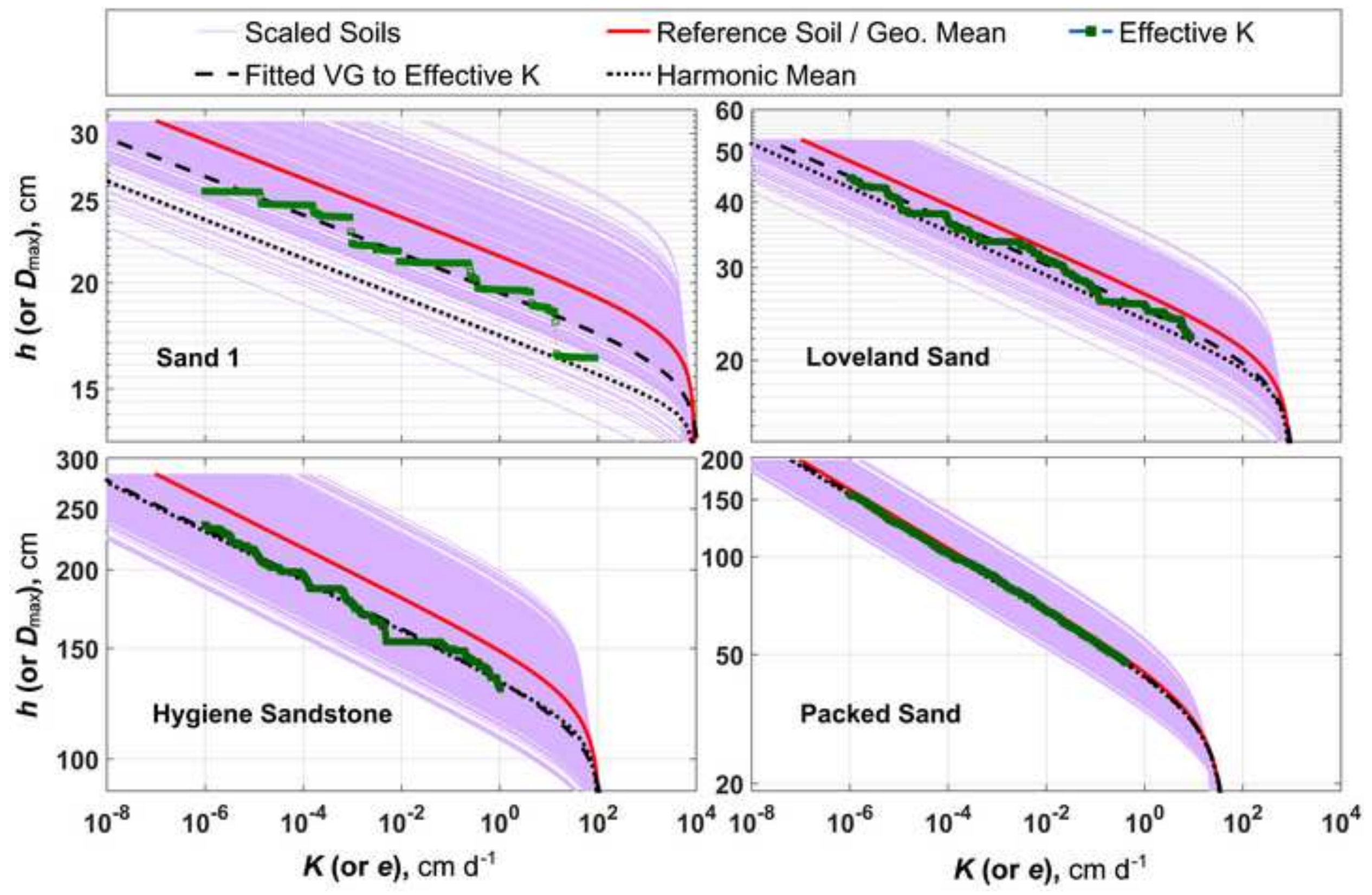



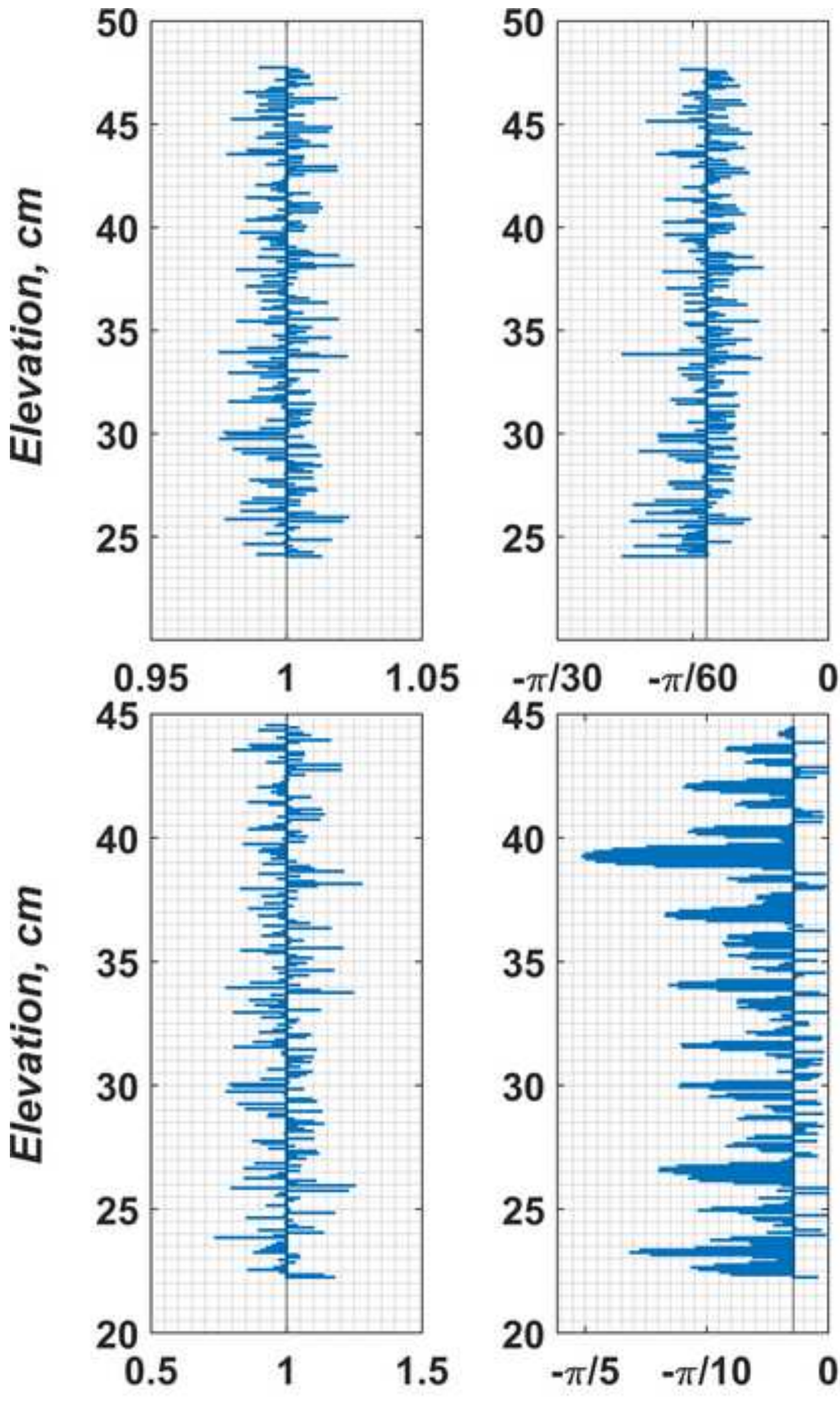

Scaling Factor $\operatorname{Arctan}(\beta)$, radians 

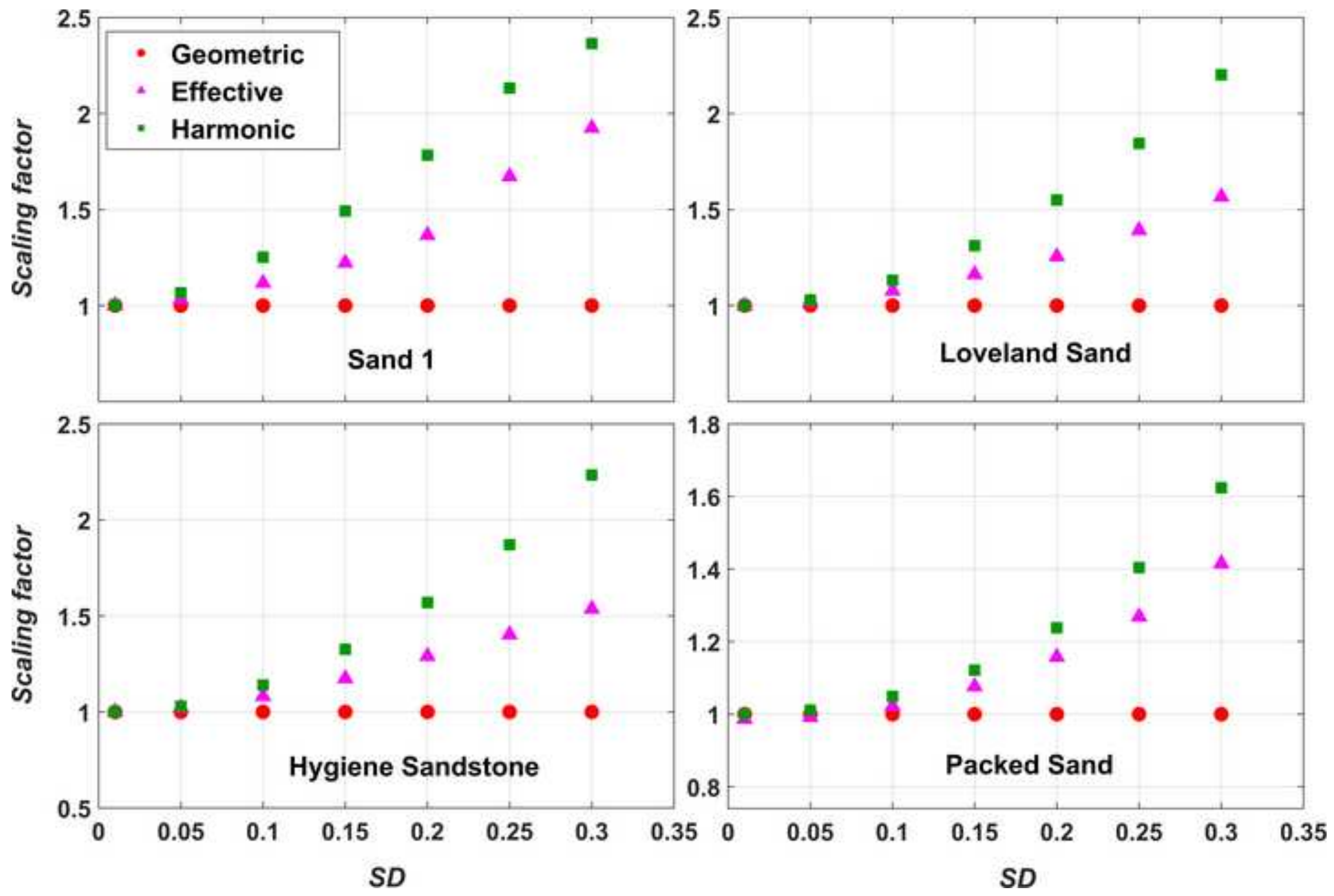


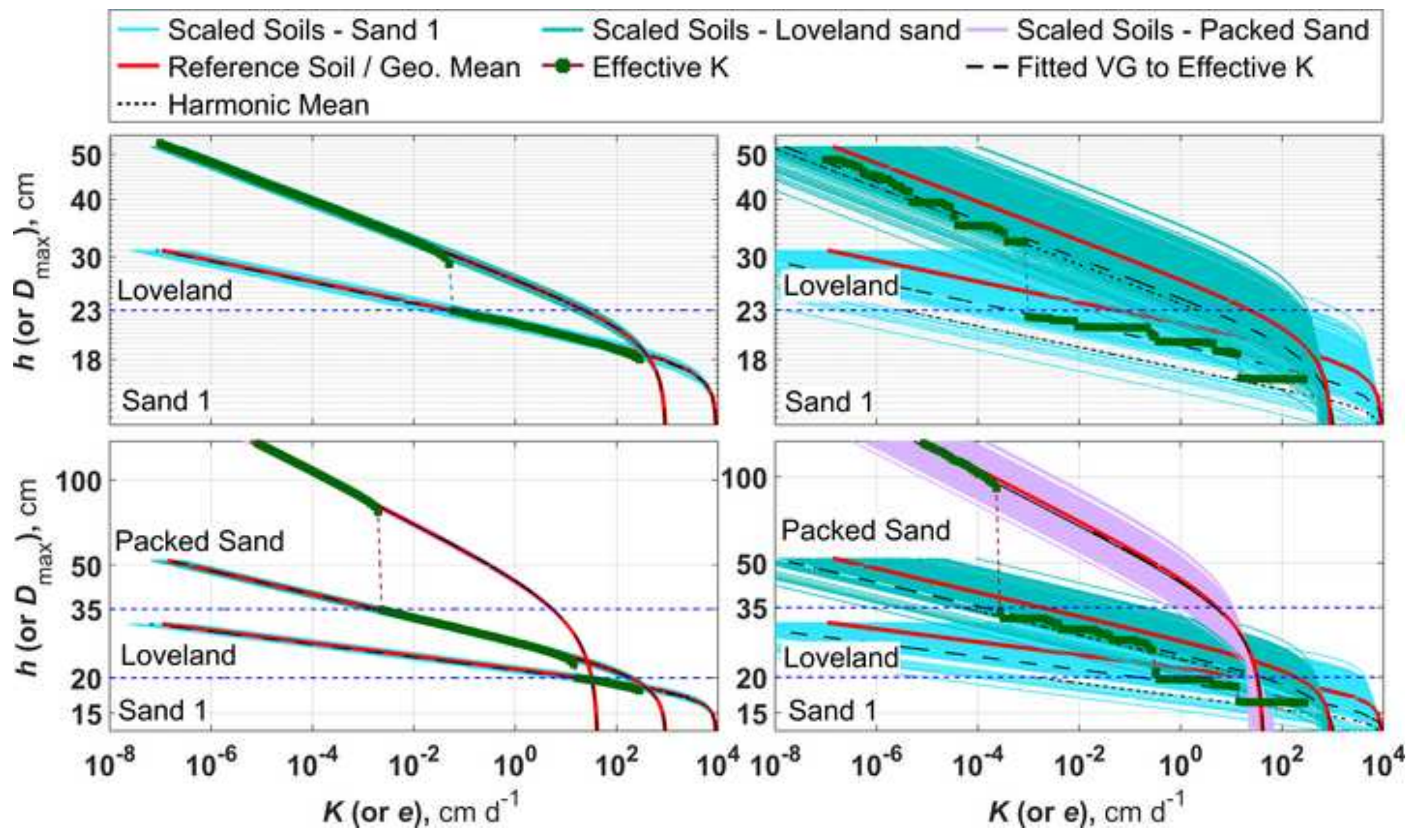



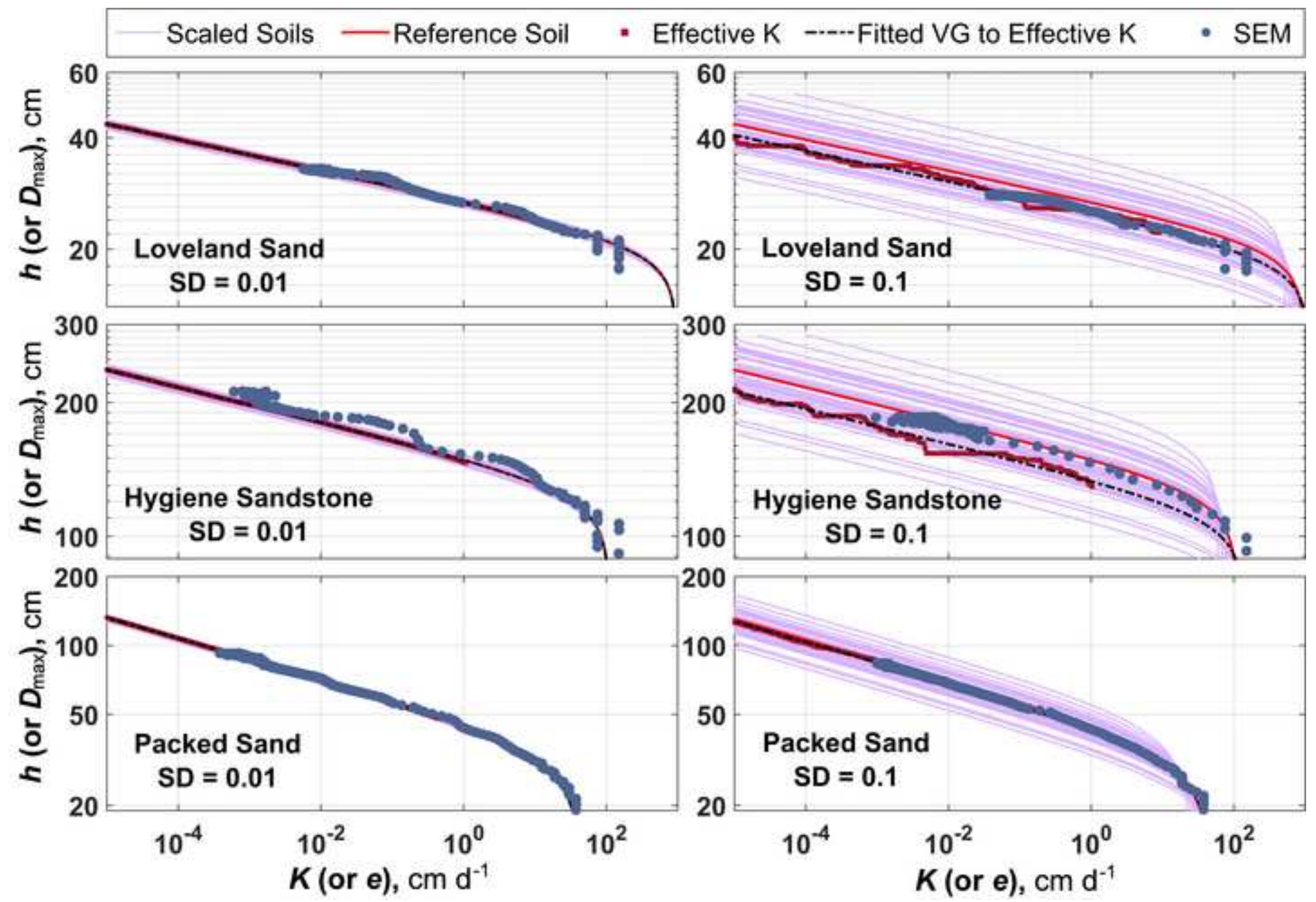


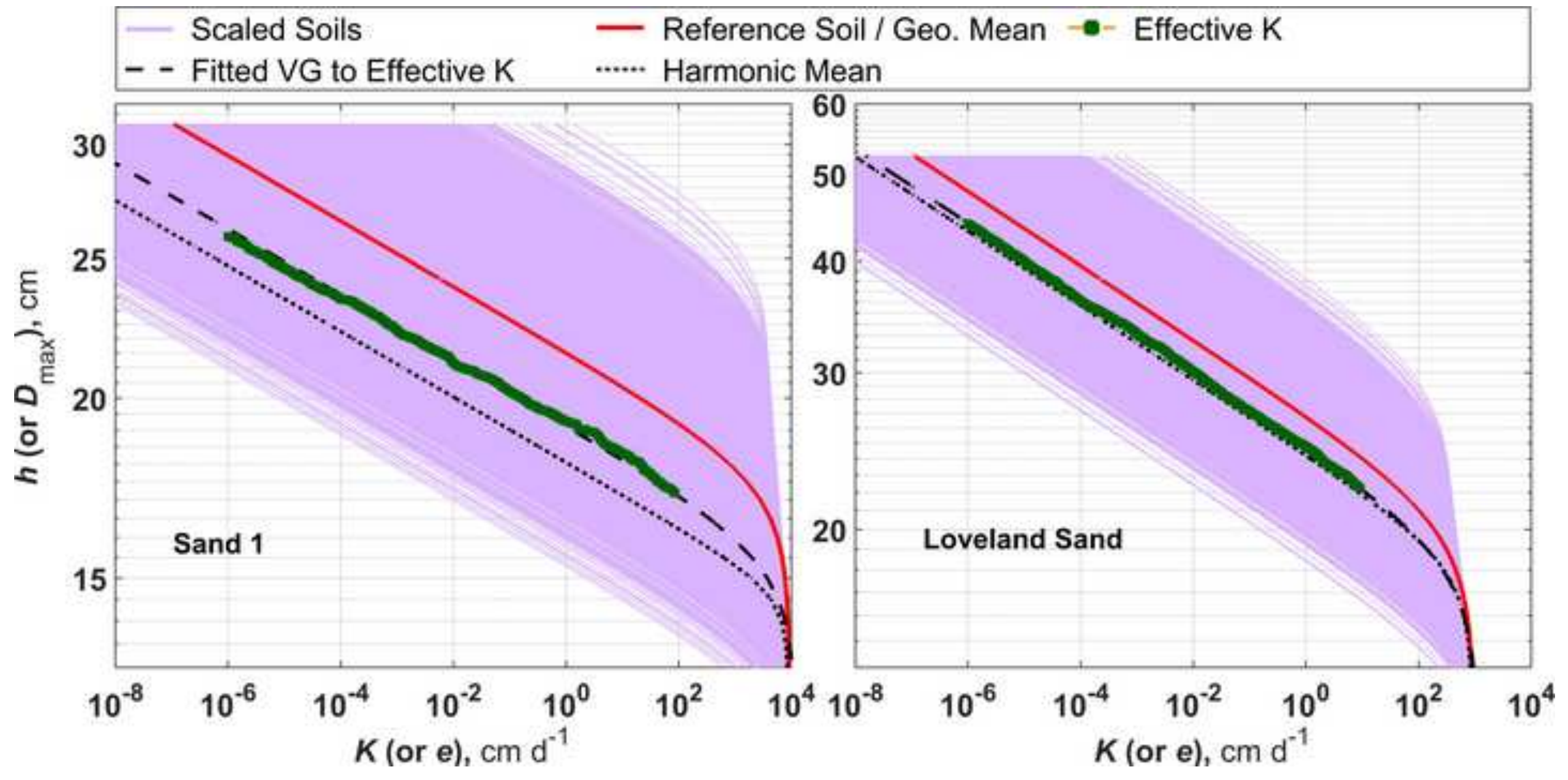


1 Table 1: van Genuchten (1980) parameters for investigated soils.

\begin{tabular}{lccc}
\hline Soil Name & $\boldsymbol{\alpha}\left(\mathrm{cm}^{-1}\right)$ & $\boldsymbol{n}$ & $\boldsymbol{K}_{\boldsymbol{s}}\left(\mathrm{cm} \mathrm{d}^{-1}\right)$ \\
\hline Sand-1 & 0.0570 & 17.80 & 9158.4 \\
Loveland Sand & 0.0490 & 9.79 & 945.3 \\
Hygiene Sandstone & 0.0079 & 10.40 & 108.0 \\
Packed Sand & 0.0290 & 4.64 & 43.7 \\
\hline
\end{tabular}

2 
1 Table 2: Pressure head, $h_{\max }$, and hydraulic conductivity, $K\left(h_{\max }\right)$, at the upper boundary of the 2 liquid domain (i.e. drying front).

\begin{tabular}{lcc}
\hline Soil Name & $\begin{array}{c}\boldsymbol{h}_{\max } \\
(\mathrm{cm})\end{array}$ & $\begin{array}{c}\boldsymbol{K}_{l}\left(\boldsymbol{h}_{\max }\right) \\
\left(\mathrm{cm} \mathrm{d}^{-1}\right)\end{array}$ \\
\hline Sand-1 & 31.63 & $4.49 \mathrm{E}-8$ \\
Loveland Sand & 53.70 & $6.42 \mathrm{E}-8$ \\
Hygiene Sandstone & 331.13 & $1.98 \mathrm{E}-9$ \\
Packed Sand & 199.92 & $9.07 \mathrm{E}-8$ \\
\hline
\end{tabular}

4 